\title{
Adult zebrafish as a model organism for behavioural genetics
}

\author{
William Norton*, Laure Bally-Cuif ${ }^{*}$
}

\begin{abstract}
Recent research has demonstrated the suitability of adult zebrafish to model some aspects of complex behaviour. Studies of reward behaviour, learning and memory, aggression, anxiety and sleep strongly suggest that conserved regulatory processes underlie behaviour in zebrafish and mammals. The isolation and molecular analysis of zebrafish behavioural mutants is now starting, allowing the identification of novel behavioural control genes. As a result of this, studies of adult zebrafish are now helping to uncover the genetic pathways and neural circuits that control vertebrate behaviour.
\end{abstract}

\section{Review}

Henry David Thoreau wrote "Many men go fishing all of their lives, without knowing it is not fish they are after". Thus, one of the intrinsic difficulties of studying behaviour is to understand the underlying motivation of complex behaviours. Most behavioural traits are multigenic and display environmental interactions, further compounding the difficulty of analysing them. However, recent studies using rats, mice, zebrafish, nematodes and fruit flies have begun to identify the genetic toolbox that controls behaviour.

The general suitability of zebrafish as a model organism, as well as its use in the genetic and neuroanatomical analysis of larval behaviour has been comprehensively described elsewhere [1,2]. Although more difficult to manipulate than larvae, adult zebrafish display a full repertoire of mature behaviours making their characterisation particularly enticing. Zebrafish (Danio rerio) are a typical cyprinid (carp family) schooling fish. In contrast to other laboratory behavioural models, zebrafish are naturally social animals that show preference for the presence of conspecifics [3,4]. Zebrafish are therefore an excellent model to probe the genetics of social behaviour. In addition, zebrafish are diurnal allowing behaviour to be measured during their natural day time. Finally, it is crucial to investigate whether complex behaviours such as reward, learning and social

\footnotetext{
* Correspondence: norton@inaf.cnrs-gifffr; bally-cuif@inaf.cnrs-gifffr Zebrafish Neurogenetics group, Laboratory of Neurobiology \& Development (NED), CNRS, UPR 3294, Institute of Neurobiology Albert Fessard, Avenue de la Terrasse, 91198 cedex, Gif-sur-Yvette, France
}

behaviour are conserved throughout the animal kingdom. Thus, comparative studies of many model organisms, including zebrafish, are necessary to determine general principles of behavioural control. Several groups have already developed protocols to measure aggression, alarm reaction, antipredatory behaviour, anxiety, locomotion, learning and memory, sleep, reward and social behaviour (see Table 1 and references therein). In this review we consider the brain areas and neurotransmitter systems that have been linked to the control of behavioural in adult zebrafish. We also describe the protocols and tools that have been developed for zebrafish behavioural studies.

\section{Contributions of zebrafish to behavioural genetics: Reward and Learning \\ Reward behaviour}

Perhaps the most prominent area in which the adult zebrafish has contributed to behavioural genetics is reward. Reward behaviour provides animals with an instinctive drive to search for resources and to reproduce. However, the brain's reward pathway can also be hijacked by drugs of abuse such as cocaine, amphetamine or opioids. Reward behaviour may thus constitute the first step towards addiction. Reward can been measured in zebrafish by using the conditioned place preference (CPP) test, which pairs a primary cue (e.g. a drug) with a secondary stimulus such as a coloured aquarium compartment. Drug dependency can also be evaluated by measuring the persistence of CPP following a period of abstinence.

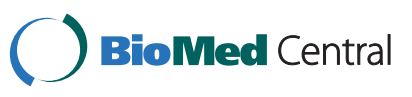


Table 1 Protocols to measure behaviour in adult zebrafish

\begin{tabular}{|c|c|c|c|}
\hline Stage & Behaviour & Paradigm & Reference \\
\hline$\overline{\text { Adult }}$ & Aggression & Live observation of two fish & {$[29,37,39,40,45]$} \\
\hline Adult & Aggression & Mirror image test & {$[6]$} \\
\hline Adult & Aggression & Pigment response & [6] \\
\hline Adult & Aggression & Startle reaction & {$[85]$} \\
\hline Adult & Alarm reaction & Response to alarm substance & {$[26,52,86]$} \\
\hline Adult & Antipredation & Predator stimulation & {$[87]$} \\
\hline Adult & Anxiety & Exit latency test & {$[51]$} \\
\hline Adult & Anxiety & Group preference & {$[6]$} \\
\hline Adult & Anxiety & Light/Dark preference & {$[48,88]$} \\
\hline Adult & Anxiety & Locomotory activity & {$[6]$} \\
\hline Adult & Anxiety & Place preference / Thigmotaxis & {$[13,34,49,51]$} \\
\hline Adult & Anxiety & Tank diving test & {$[50,53,55]$} \\
\hline Adult & Anxiety & Time in enriched T-maze chamber & {$[89]$} \\
\hline Adult & Audition & Response to startling noise & [64] \\
\hline Adult & Courtship & Observation of courtship postures & {$[82]$} \\
\hline Adult & Lateralisation & Interaction with object & {$[41,77]$} \\
\hline Adult & Locomotion & Mean velocity & {$[90]$} \\
\hline Adult & Locomotion & Number of lines crossed & {$[36,81,87]$} \\
\hline Adult & Locomotion & Total distance moved / Videotracking & {$[80,81,90]$} \\
\hline Adult & Locomotion & Turning angle & {$[90]$} \\
\hline Adult & Mate choice & Video-stimulus technique & {$[91]$} \\
\hline Adult & Learning / memory & Active avoidance conditioning & {$[27,28,92,93]$} \\
\hline Adult & Learning / memory & Delayed spatial alternation & {$[35]$} \\
\hline Adult & Learning / memory & Learned alarm reactions & {$[86]$} \\
\hline Adult & Learning / memory & Spatial alternation, learning and memory & {$[31,32,54,94]$} \\
\hline Adult & Learning / memory & T-maze & {$[8,13,33,89]$} \\
\hline Adult & Learning / memory & Visual discrimination learning & {$[29]$} \\
\hline Adult & Olfaction & Response to amino acids & {$[75]$} \\
\hline Adult & Reward & Conditioned place preference & {$[6-9,13]$} \\
\hline Adult & Reward & Presence of Conspecific & {$[11]$} \\
\hline Adult & Sleep & Locomotor inhibition & {$[61]$} \\
\hline Adult & Sleep & Monitoring sleep postures & {$[57,58,63]$} \\
\hline Adult & Sleep & Pigment response & {$[61]$} \\
\hline Adult & Social preference & Area occupied & {$[85]$} \\
\hline Adult & Social preference & Group preference & {$[6]$} \\
\hline Adult & Social preference & Nearest neighbour distance & {$[85]$} \\
\hline Adult & Social preference & Shoaling & {$[3,78,87]$} \\
\hline Adult & Vision & Optokinetic response & {$[76]$} \\
\hline
\end{tabular}

In line with studies of other animals (e.g. [5]), stimuli that have been shown to be rewarding for adult fish include ethanol [6,7], cocaine [8], amphetamine [9], opiates [10], nicotine [7], food [10] and the presence of conspecifics [11]. The major neurotransmitter associated with reward behaviour is Dopamine (DA). Increases of DAergic signalling from the ventral tegmental area to the nucleus accumbens ( $\mathrm{nAC}$ ) motivates mammals to repeat stimulus application. In zebrafish, this key DAergic pathway is most likely comprised of projections from the diencephalic posterior tuberculum to the ventral telencephalon (subpallium, ( $\mathrm{Vv}$ and $\mathrm{Vd})$, see [12]).
Several other neurotransmitters have also been implicated in reward behaviour. Heterozygous mutant zebrafish lacking one copy of the acetylcholinesterase (ache) gene have enhanced acetylcholine levels in the brain due to decreased breakdown of the neurotransmitter. The increase of acetylcholine in the brain of ache mutants causes a decrease in amphetamine-induced CPP [13]. Mammalian reward pathways also include raphe 5-HTergic neurons [14] as well as a number of inhibitory influences including projections from the habenula. The zebrafish ventral habenula appears to be homologous to the mammalian lateral habenula in both gene 
expression and innervation of the raphe [15]. The recent identification of selective molecular markers for both structures $[16,17]$ will make genetic manipulation of the reward pathway possible. Such a targeted approach will allow functional interrogation of the reward circuitry in zebrafish and may highlight both similarities and differences in the mechanisms controlling monoaminergic behaviours in vertebrates.

There have been several screens for zebrafish mutant families with altered reward behaviour. Darland and Dowling isolated three families of mutants which were not responsive to cocaine application, although the affected genes were not reported [8]. Other groups have used microarrays to identify addiction related genes. Brennan and colleagues demonstrated a robust change in place preference (PP) following nicotine or ethanol treatment [7]. This PP was also maintained following a period of abstinence or when paired with an adverse stimulus (3 seconds removal from the tank water) suggesting that drug dependency had occurred. Microarray analysis comparing the brains of both treated and untreated fish identified 1362 genes that were significantly changed following drug application, including 153 that were responsive to both nicotine and ethanol. Many of these genes are also involved in reward behaviour in other species, by either altering dopaminergic or glutamatergic signalling or modulating synaptic plasticity [7]. In addition, this study also revealed a number of novel genes that were changed upon drug administration, including those coding for Calcineurin B and the Hypocretin receptor. Bally-Cuif and colleagues conducted a screen for mutants that were insensitive to amphetamine application [13]. One of these mutants, no addiction (nad), was used to identify genes that were transcriptionally modified by amphetamine in wild-type fish and were differentially over- or under-regulated in nad. Importantly, gene expression was unmodified in nad mutants in the absence of the drug [9]. This strategy permitted the unbiased recovery of 139 genes linked to amphetamine triggered CPP. A large proportion these genes were developmentally active transcription factors. These include Dlx1a, Emx1a, Lhx8, Sox9 and Tbr1, proteins that are implicated in the control of neurogenesis in the vertebrate embryo and show persistent expression in the adult-neurogenic regions of the mammalian and fish brain [9]. A recent study of rats has also demonstrated altered cocaine (but not sucrose) mediated reward behaviour following a reduction of hippocampal neurogenesis [18]. This constitutes an exciting new development in the field of reward behaviour; neurogenesis-induced plasticity may account for some of the learning aspects of reward and the long-lasting changes in the brain associated with addiction.
A comparable approach has led to the identification of too few (tof) a mutant that fails to change place preference following morphine treatment but not food application [10]. tof encodes a forebrain specific zinc finger protein, Fezl [19], which establishes neurog1-expressing DA progenitor domains in the basal forebrain [20]. Loss of $f e z l$ leads to a reduction of dopaminergic and serotonergic neurons in specific nuclei of the forebrain (diencephalon and hypothalamus; [21]), defects that are maintained into adulthood. Dissociation between the preference for a natural reward (food) and a drug (morphine) has previously been observed in dopamine D2 receptor knock-out mice [22] but is not understood at the molecular or neurological level. Since both morphine and food rewards are dependent on opioid receptor activity in zebrafish [10], the separable reward behaviour seen in tof suggests that distinct neural systems act downstream of opioid signalling to mediate the response to morphine and food. Alternatively, the rewarding aspects of food and drug treatment may be mediated by different subsets of dopaminergic nuclei in the forebrain. Thus, together with the study of hippocampal irradiated rats [18], tof presents an excellent opportunity to dissect the neural basis of discrimination between rewarding substances in the brain.

\section{Learning and memory}

Studies of mammals have shown that learning and memory can be controlled by several brain circuits, each of which is neuroanatomically distinct. These include spatial learning (hippocampus), implicit learning (such as simple motor reflexes; cerebellum) and avoidance learning (amygdala). Although the neural basis of learning is not well understood in zebrafish, studies of the closely related goldfish (Cassius auratus) hint at brain areas which could be involved. Focal ablations of the goldfish brain have identified the lateral pallium (Dl, equivalent to the hippocampus), medial pallium (Dm, equivalent to the amygdala) and cerebellum [23-25] as playing key roles in learning.

Several paradigms have already been developed to measure learning and memory in zebrafish. Associative learning can be measured by pairing two previously unrelated stimuli such as colour, reward or aversion. For example, Suboski and colleagues paired a neutral stimulus (morpholine) with the aversive effects of alarm substance [26]. Avoidance learning can be assessed by using a shuttle box; fish are quickly able to associate a conditioned stimulus (e.g light [27] or colour [28]) with an unconditioned stimulus (such as a mild electric shock). Spatial learning can be measured using either a T-maze $[8,11,29,30]$ or a shuttle box $[31,32]$. Fish must learn to collect a reward by either navigating a maze correctly or alternating the side of the tank visited. These behavioural tests are high-throughput, making them suitable 
for screens for novel genes controlling learning and memory.

Pharmacological studies have validated adult zebrafish as a model for learning and memory, making it a very promising area for future research. Several evolutionarily conserved neurotransmitter systems have been implicated in learning and memory. ache mutants, with increased acetylcholine levels in the brain, learn to find a food reward faster in a T-maze [33] thereby linking cholinergic signalling to learning. Fish exposed to moderate levels of nicotine perform better in a delayed spatial alteration task, a type of avoidance learning test. Nicotine acts via nicotinic acetylcholine receptors (nACHRs), thus further underscoring the importance of cholinergic signalling $[34,35]$. Treatment of fish with a Histidine decarboxylase inhibitor, alphafluoromethylhistidine $(\alpha-F M H)$, reduces both levels of histamine and the number of histaminergic fibres in the brain. $\alpha-\mathrm{FMH}$ treated animals display defects in long-term memory formation but not initial learning [30]. Finally, NMDA antagonists have also been shown to impair memory formation in zebrafish $[27,28]$. NMDA receptors are found abundantly in the telencephalon, which contains the teleostean (bony fish) equivalent of the hippocampus and amygdala [36]. The neurotransmitters discussed above have also been connected to learning in other species, suggesting that work in zebrafish may give insight into conserved learning mechanisms. Therefore, adult zebrafish constitute a particularly promising model for research into learning and memory.

\section{Emerging fields for genetic analysis: Aggression and Anxiety Aggression}

Aggression is a complex suite of behaviours serving a number of adaptive purposes. Fish use aggression to protect offspring, monopolise resources such as food, territory and mates and establish dominance hierarchies. Aggression can be measured in the laboratory by recording the interaction of two free-swimming fish or by using mirror induced stimulation (MIS) $[6,37]$. Fish are unable to recognise their own image and so attack as if an intruder is present [38]. Furthermore, MIS provides immediate feedback to the fish's activity and avoids damaging the subjects [37]. Zebrafish display characteristic agonistic postures including erection of the dorsal, caudal, pectoral and anal fins coupled to biting, thrashing of the tail and short bouts of fast swimming directed against the mirror [6]. A positive correlation between aggression and boldness has also been reported [39]. Aggression is a very plastic behaviour. Both habitat complexity and rearing conditions can influence the number of interactions $[37,40]$. Furthermore, different wild-type strains show varying aggression levels suggesting a genetic component to its control. Finally, aggressive behaviour also shows lateralisation, with adult fish predominantly using the right eye to view predators [41].

Studies in other species have identified 5-HT as the major neurotransmitter controlling aggression. Animals with high levels of $5-\mathrm{HT}$ tend to be timid, whereas those with lower levels are more impulsive and aggressive (e.g. [42]). Other neurotransmitters, including GABA, glutamate and nitric oxide as well as the hormones vasopressin and testosterone have also been implicated in agonistic behaviour [43]. However, the role of these neurotransmitters has not been explicitly tested in zebrafish. In zebrafish, agonistic behaviour can be modified by exposure to pharmacological compounds including ethanol [6] and 17alpha-ethinylestradiol (a synthetic oestrogen; [44]). The brain areas mediating aggression in fish are not well characterised. Arginine vasotocin-expressing cells of the magnocellular preoptic area change size depending on the dominance status of fish. This suggests involvement of the preoptic area in control of social hierarchy and the agonistic behaviour used to establish it [45]. Studies of other fish species have identified additional brain territories that influence aggression. For example, the neural activity maker cfos is expressed in the diencephalon, thalamus and hypothalamus and a few nuclei in the pons and medulla oblongata of the mudskipper (Periophthalmus cantonensis) following an aggressive episode [46]. Finally, electrical stimulation of the bluegill (Lepomis macrochirus) implicates the inferior hypothalamus in aggression control [47].

The MIS protocol is simple to establish and perform. Coupled to computer-aided automation, it can be adapted for high-throughput screening studies, thus providing a golden opportunity to uncover novel genes implicated in aggression control.

\section{Anxiety}

Anxiety is a state of constant fear or restlessness caused by anticipation of a real or imagined future event. Multiple anxiety tests have been established in fish, although it is not always clear whether fear or anxiety is being measured, or indeed whether the different states even exist [48]. Protocols to measure anxiety tend to assess one of two variables. The first set of protocols record the reaction of adult fish to novel environments, such as the amount of time spent at the edge of a tank $[30,49]$, at the bottom of a novel tank $[34,50]$ or on the dark side of a light/dark tank [28,51]. The second approach analyses locomotory patterns: freezing, long-lasting increases in basal locomotory activity $[6,48,49]$ and tightening of a fish's shoal [52] have all been reported to be reliable measures of anxiety. The expression and level of anxiety are wild-type strain dependent $[49,50]$. 
For example, $\mathrm{AB}$ wild-types manifest anxiety as a hyperactive swimming response [49].

Similar to other behaviours, anxiety protocols have been validated using pharmacological compounds developed for human patients. Application of caffeine [50,53], pentylenetetrazole [53], alarm substance [50,52], the benzodiazepine partial inverse agonist FG-7142 [49] and withdrawal of cocaine [49] have all been shown to be anxiogenic. Conversely, many anxiolytic substances have been characterised including nicotine [54], diazepam $[49,55]$, the Htr1a (5-HT receptor) partial agonist buspirone [55], fluoxetine hydrochloride and ethanol [50]. Finally, a link between anxiety levels and the major zebrafish stress hormone cortisol has also been demonstrated [50]. The ease of applying drugs and robust behavioural assays (see $[49,50]$ ) make zebrafish an ideal model to study anxiety and related behaviours.

\section{Sleep}

Although sleep is a widespread phenomenon, its behavioural and physiological function is not well understood. Sleep is characterised by periods of behavioural quietness, species-specific body postures, an increased arousal threshold and a quick return to wakefulness [56]. Furthermore, sleep-deprived animals also show homeostatic rebound, increasing the amount of time needed to sleep following deprivation. The timing of sleep also shows circadian rhythmicity. Several studies have identified sleep-like behaviour in zebrafish. During the night, adult fish have periods of two to four minutes of inactivity in which the fish floats horizontally and makes small pectoral fin movements. There is also a simultaneous reduction of mouth and operculum movements suggesting lower respiratory levels [57]. Sleep rebound has been demonstrated in zebrafish indicating homeostatic regulation; disrupting the normal night time routine (using light, vibration, electric shock or forced movement) deprives fish of rest and causes a subsequent increase in sleep duration $[57,58]$. Finally, zebrafish also show circadian rhythmicity, with higher activity levels in the day [57].

Studies in other species have identified several significant sleep-related neurotransmitters: Increases of dopamine levels in the brain reduces the amount of time asleep [59], whereas GABA signalling promotes sleep and $\mathrm{GABA}_{\mathrm{A}}$ receptor agonists are used to treat insomnia [60]. Although these pathways have not been directly examined in zebrafish, treatment with diazepam, pentobarbital [57], alpha2 adrenoceptor agonists [61], and histamine $\mathrm{H} 1$ antagonists [62] have all been shown to increase sleep, thus implicating GABA, acetylcholine and histamine in its control. Several studies have also demonstrated a conserved role for hypocretin/orexin $(\mathrm{HCRT})$ in sleep-wake regulation. Zebrafish contain a single HCRT receptor gene (hcrtr), which is expressed in a small number of glutamatergic neurons of the adult hypothalamus $[58,63]$. Loss of hcrtr function causes sleep fragmentation but not cataplexy or decreased wake bout length, suggesting that HCRT may function to consolidate sleep in fish [58]. HCRT acts by stimulating the endogenous melatonin sleep-promoting system found in the pineal gland [63]. Taken together, studies of zebrafish have confirmed that the control of sleep appears to be evolutionarily conserved. Although zebrafish sleep research is still in its infancy, the high throughput nature of the set-ups used to measure sleep demonstrates that zebrafish are an ideal model in which to conduct screens for novel hypnotic mutants.

\section{Practical considerations: strain differences, screen design and duplicated genes Strain differences in wild-type fish}

The examples discussed in this review highlight the suitability of adult zebrafish for studies of complex vertebrate behaviours. However, there are several considerations that need to be taken into account before initiating behavioural work. For example, care must be taken to dissect the influence of neurotransmitter signalling pathways and the specificity of drugs used to modulate them. Finally, another important consideration when designing behavioural studies is the background strain of the fish used. Strain differences in adult behaviour have already been reported [13,49,50,64]. Thus, in order to avoid some of the known difficulties in reproducing behavioural work, all behavioural studies should be carried out on well defined laboratory strains. Although no inbred strains exist, the $\mathrm{AB}$ line, available from the ZIRC stock centre is an excellent choice for a reference strain. The line has been maintained in the laboratory for more than 70 generations and is freely available to the zebrafish community.

\section{Screen design}

Genes do not directly control behaviour. Rather, genes influence behavioural output by either modulating neural circuit formation (neural specification, differentiation and connectivity) or function (e.g. neurotransmitter release or reuptake). High throughput forward genetic screening has long been one of the goals of zebrafish research, and in this regard the nascent behavioural field is no different. However, behavioural phenotyping is subject to large variability between animals. This can make it difficult to phenotype mutants with certainty, and so complicates positional cloning of the mutations. Furthermore, careful consideration needs to be given to the choice of mutagen. The most commonly used mutagen N-ethyl-N-nitrosourea (ENU; Fig. 1) efficiently induces intragenic point mutations in the germline [65], but the subsequent cloning steps needed to recover the mutagenised gene are 


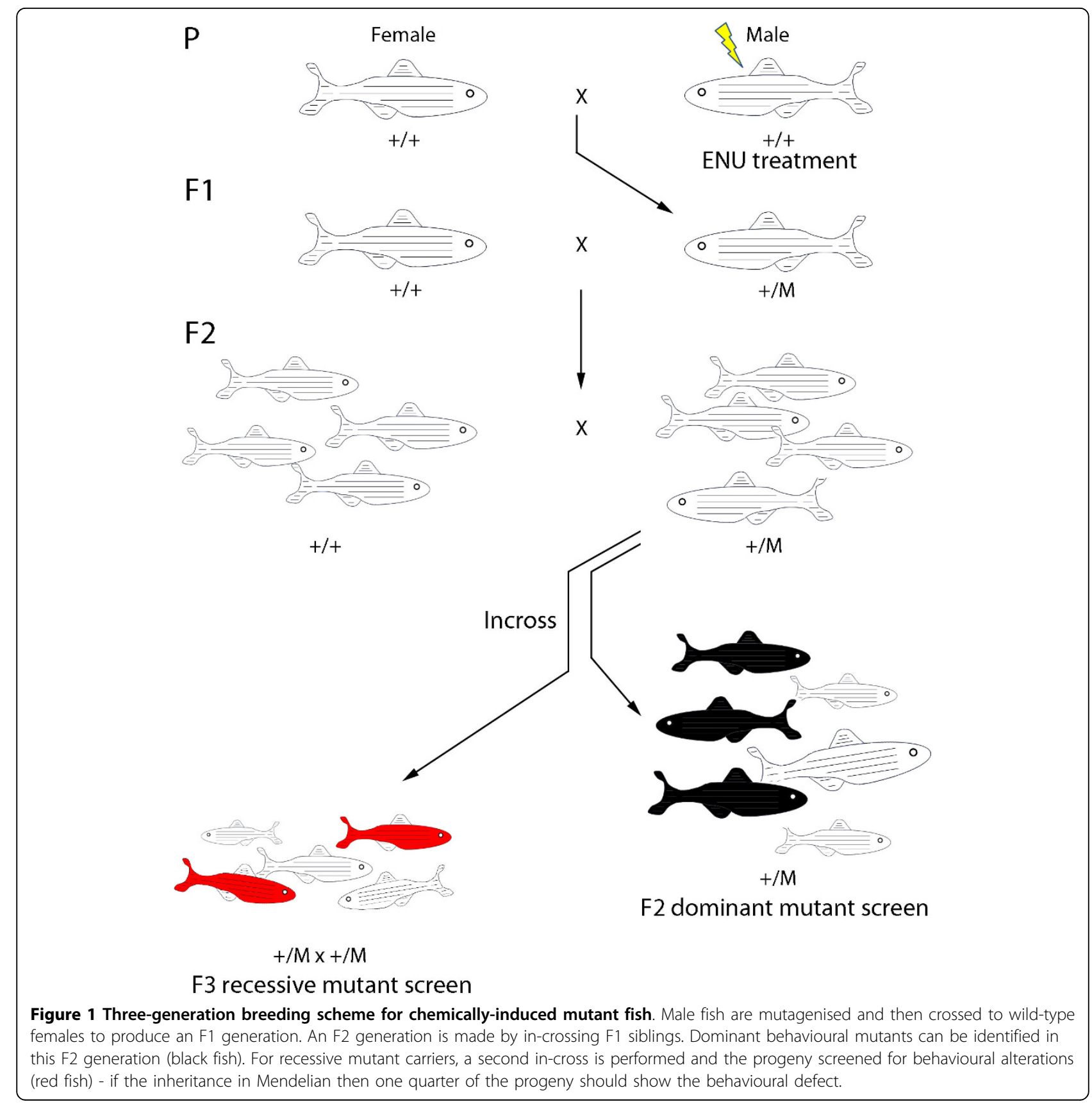

laborious. As an alternative to ENU treatment, insertional mutagenesis looks particularly promising (Fig 2). Although insertional mutations occur at a lower frequency, isolation of the genetic lesion is much simpler $[66,67]$. The mutagenic cassette may also be coupled to a fluorescent reporter line thus highlighting the expression profile of the mutated gene. This technique will allow faster and more reliable identification of animals carrying the same insertion and so will facilitate mapping. This has recently been powerfully demonstrated in juvenile fish by using a reporter-tagged insertional mutagenesis strategy to clone two nicotine-response mutants [68]. Finally, the usefulness of zebrafish is not limited to screening paradigms. The advent of TILLING [69] and zinc-finger nuclease technology [70] has opened the door to targeted modification of the zebrafish genome, thus allowing the behavioural function of known genes to be probed.

\section{Gene duplication and redundancy in zebrafish}

In common with all ray-finned fishes (actinopterygii), zebrafish underwent a third whole genome duplication 


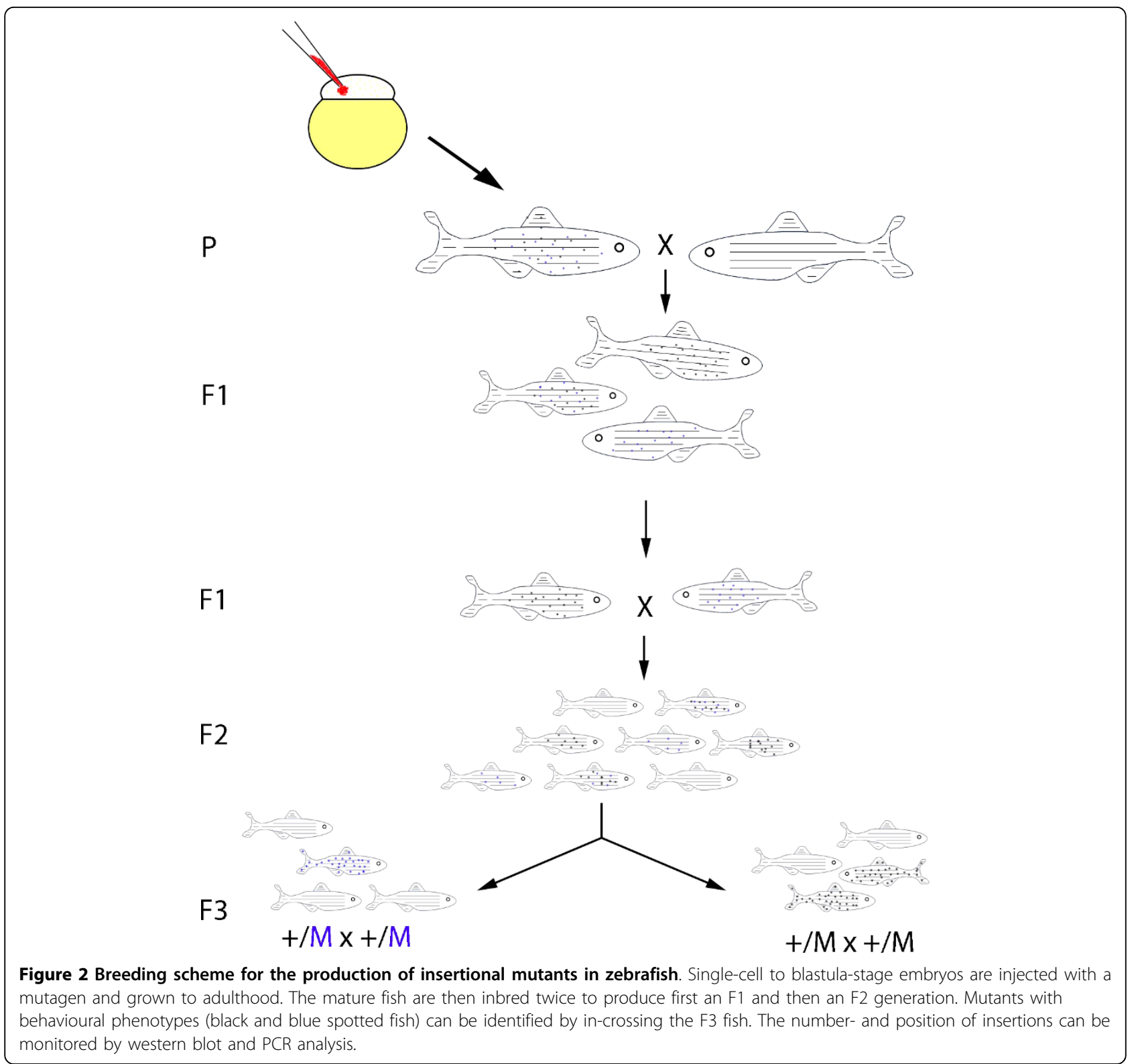

around 350 million years ago and often have two copies of genes found in other vertebrates [71]. The most likely fate of a duplicate gene is loss of function. However, in some cases both copies can be retained and subfunctionalisation (splitting of the ancestral function between the two new genes) or neofunctionalisation (acquisition of a new function through mutation) can occur [72]. Redundancy can make analysis of a gene's function more difficult by masking mutant phenotypes. However, redundancy can also be useful, exposing late functions of genes that cause embryonic defects in other animals. For example, zebrafish lacking activity of one copy of fibroblast growth factor 1 (fgfrla) have a surprising lack of developmental phenotype compared to mice and medaka deficient in the gene $[73,74]$. Rather, adult fgfrla mutant fish exhibit several behavioural alterations, including increases in aggression, boldness and exploration (W Norton, personal observation).

\section{Conclusion}

Although anecdotally fish are thought to have poor memories and display few complex behaviours, numerous studies have disproved such beliefs. In this review we have demonstrated ways in which studies of adult zebrafish have contributed to our understanding of the genetic basis of behaviour. We have described set-ups to measure behaviour (e.g. Table 1) and some of the pharmacological treatments that have already been employed 
in zebrafish (Table 2). However, fish also manifest other behaviours, the discussion of which is unfortunately beyond the scope of this review. These behaviours include olfaction [75], vision [76], behavioural lateralisation [77], shoaling [3,78,79], locomotion [80,81] and reproductive behaviour [82]. Finally, studies of adult fish are also beginning to give clues about the initiation of locomotion, an assay that might be modified to probe the motivation to move. In the adult spinal cord, application of 5-HT modifies the cyclical pattern of locomotory activity by increasing mid-cycle inhibition and reducing the onset of the next cycle, so reducing the initiation of locomotion [83].

Larval zebrafish are also useful for studying simple behaviours, and protocols have been established to measure locomotion and visuomotor behaviours such as prey capture [1]. Coupled to the transgenic lines available and the emergence of optogenetic technology (e.g. [84]), larvae may allow the dissection of behavioural circuits at the cellular level in intact living fish. Moreover, in an elegant recent study by Engert and colleagues, neural circuit activity has been analysed at the singlecell level by recording bioluminescence in free-swimming larvae [85].

In summary, zebrafish have many attributes that make them an ideal model organism for the study of behavioural genetics. Although to date there have been relatively few studies of adult zebrafish behaviour, the ease of carrying out pharmacological studies coupled to the ever increasing number of available genetic tools suggest

Table 2 Pharmacological treatments with known behavioural effects on adult zebrafish

\begin{tabular}{|c|c|c|c|c|}
\hline Behaviour & Modulating agent & Function / Activity & Effect & Reference \\
\hline Aggression & Ethanol & GABA-A receptor modulator & Increases aggression & {$[85]$} \\
\hline Aggression & $17 \alpha$-ethinylestradiol & Synthetic oestrogen & Reduces aggression & {$[44]$} \\
\hline Antipredation & Ethanol & GABA-A receptor modulator & Impaired by high doses & {$[6]$} \\
\hline Anxiety & Diazepam & Benzodiazepine & Reduces anxiety & {$[49,86]$} \\
\hline Anxiety & FG-7142 & Benzodiazepine inv. agonist & Increases anxiety & {$[49]$} \\
\hline Anxiety & Pentylenetetrazole & GABA antagonist & Increases anxiety & {$[53]$} \\
\hline Anxiety & Ethanol & GABA-A receptor modulator & Reduces anxiety & {$[50,53]$} \\
\hline Anxiety & Buspirone & Htr1A partial agonist & Reduces anxiety & {$[55]$} \\
\hline Anxiety & Alarm substance & Hypoxanthine-3N-oxide & Increases anxiety & {$[50,52]$} \\
\hline Anxiety & Nicotine & NachR agonist & Reduces anxiety & {$[34]$} \\
\hline Anxiety & Methyllycaconitine & Nicotinic antagonist & Anxiolytic & {$[55]$} \\
\hline Anxiety & Dihydro- $\beta$-erythroidine & Nicotinic antagonist & Anxiolytic & {$[55]$} \\
\hline Anxiety & Mecamylamine & Nicotinic antagonist & Anxiolytic & {$[34]$} \\
\hline Anxiety & Morphine & Opiate & Reduces anxiety & {$[53]$} \\
\hline Anxiety & Cocaine (withdrawal) & Psychostimulant & Increases anxiety & {$[49]$} \\
\hline Anxiety & Fluoxetine & 5-HT reuptake inhibitor & Reduces anxiety & {$[50,53]$} \\
\hline Anxiety & Caffeine & Xanthine alkaloid & Increases anxiety & {$[50,53]$} \\
\hline Group preference & Ethanol & GABA-A receptor modulator & Reduced at high conc. & {$[6]$} \\
\hline Learning & $\alpha \mathrm{FMH}$ & HDAC inhibitor & Impairs long term memory & {$[30]$} \\
\hline Learning & Nicotine & NachR agonist & Improves learning & {$[34,35]$} \\
\hline Learning & MK-801 & NMDA antagonist & Impairs memory & {$[27,28]$} \\
\hline Learning & L-NAME & NO synthase inhibitor & Impairs memory retention & {$[27]$} \\
\hline Light/Dark pref & Ethanol & GABA-A receptor modulator & Decreased at high conc. & {$[6]$} \\
\hline Locomotion & Ethanol & GABA-A receptor modulator & Reduced at high conc. & {$[6]$} \\
\hline Reward & Acetylcholine & Cholinergic agonist & Non-rewarding & {$[13]$} \\
\hline Reward & Ethanol & GABA-A receptor modulator & Rewarding & {$[7]$} \\
\hline Reward & Nicotine & NachR agonist & Rewarding & {$[7]$} \\
\hline Reward & Food & Nourishment & Rewarding & {$[10]$} \\
\hline Reward & Morphine & Opiate & Rewarding & {$[10]$} \\
\hline Reward & Morphine & Opiate & Rewarding & {$[10]$} \\
\hline Reward & Cocaine & Psychostimulant & Rewarding & {$[8]$} \\
\hline Reward & Amphetamine & Psychostimulant & Rewarding & {$[9]$} \\
\hline Sleep & Dexmedetomidine & alpha2 adrenoceptor agonist & Sedative & {$[61]$} \\
\hline Sleep & Pentobarbital & Barbiturate & Hypnotic & {$[57]$} \\
\hline Sleep & Diazepam & Benzodiazepine & Hypnotic & {$[57]$} \\
\hline
\end{tabular}


that zebrafish are about to enter the limelight. Finally, their small size and cheap maintenance costs suggest that zebrafish are ideally suited for large-scale behavioural screens. We look forwards to the next steps in the establishment of this fascinating field.

\section{Acknowledgements}

We thank Christina Lillesaar, Ina Rothenaigner, Marion Coolen and Philippe Vernier for critically reading an early version of this manuscript, and all members of the Bally-Cuif laboratory for their enthusiastic discussions about zebrafish development and behaviour.

\section{Authors' contributions}

WN conceived the review and wrote the manuscript. LB-C suggested subject areas to include in the review and worked on the manuscript. All authors read and approved the final manuscript.

Received: 27 July 2010 Accepted: 2 August 2010

Published: 2 August 2010

\section{References}

1. Fero K, Yokogawa T, Burgess HA: The behavioural repertoire of larval zebrafish. Zebrafish Models in Neurobehavioral Research Cambridge University PressKalueff AV, Cachat JM 2010.

2. Westerfield $\mathrm{M}$ : The zebrafish book. A guide for the laboratory use of zebrafish (Danio rerio). Univ of Oregon Press, Eugene, 42000.

3. Saverino C, Gerlai R: The social zebrafish: behavioral responses to conspecific, heterospecific, and computer animated fish. Behav Brain Res 2008, 191:77-87.

4. McCann LI, Koehn DJ, Kline NJ: The effects of body size and body markings on nonpolarized schooling behavior of zebra fish (Brachydanio rerio). J Psychol 1971, 79:71-75.

5. Gianoulakis C: Endogenous opioids and addiction to alcohol and other drugs of abuse. Curr Top Med Chem 2009, 9:999-1015.

6. Gerlai R, Lahav M, Guo S, Rosenthal A: Drinks like a fish: zebra fish (Danio rerio) as a behavior genetic model to study alcohol effects. Pharmacol Biochem Behav 2000, 67:773-782.

7. Kily LJ, Cowe YC, Hussain O, Patel S, McElwaine S, Cotter FE, Brennan CH: Gene expression changes in a zebrafish model of drug dependency suggest conservation of neuro-adaptation pathways. J Exp Biol 2008, 211:1623-1634.

8. Darland T, Dowling JE: Behavioral screening for cocaine sensitivity in mutagenized zebrafish. Proc Natl Acad Sci USA 2001, 98:11691-11696.

9. Webb KJ, Norton WH, Trumbach D, Meijer AH, Ninkovic J, Topp S, Heck D, Marr C, Wurst W, Theis FJ, et al: Zebrafish reward mutants reveal novel transcripts mediating the behavioral effects of amphetamine. Genome Biol 2009, 10:R81.

10. Lau B, Bretaud S, Huang Y, Lin E, Guo S: Dissociation of food and opiate preference by a genetic mutation in zebrafish. Genes Brain Behav 2006, 5:497-505.

11. Al-Imari L, Gerlai R: Sight of conspecifics as reward in associative learning in zebrafish (Danio rerio). Behav Brain Res 2008, 189:216-219.

12. Rink E, Wullimann MF: Connections of the ventral telencephalon and tyrosine hydroxylase distribution in the zebrafish brain (Danio rerio) lead to identification of an ascending dopaminergic system in a teleost. Brain Res Bull 2002, 57:385-387.

13. Ninkovic J, Bally-Cuif $L$ : The zebrafish as a model system for assessing the reinforcing properties of drugs of abuse. Methods 2006, 39:262-274.

14. Kranz GS, Kasper S, Lanzenberger R: Reward and the Serotonergic System. Neuroscience 2010, 166:1023-1035.

15. Amo R, Aizawa H, Takahoko M, Kobayashi M, Takahashi R, Aoki T, Okamoto $\mathrm{H}$ : Identification of the zebrafish ventral habenula as a homolog of the mammalian lateral habenula. J Neurosci 2009, 30:1566-1574.

16. Amo R, Aizawa H, Takahoko M, Kobayashi M, Takahashi R, Aoki T, Okamoto $\mathrm{H}$ : Identification of the zebrafish ventral habenula as a homolog of the mammalian lateral habenula. J Neurosci 2010 , 30:1566-1574

17. Lillesaar C, Stigloher C, Tannhauser B, Wullimann MF, Bally-Cuif L: Axonal projections originating from raphe serotonergic neurons in the developing and adult zebrafish, Danio rerio, using transgenics to visualize raphe-specific pet1 expression. J Comp Neurol 2009, 512:158-182.

18. Noonan MA, Bulin SE, Fuller DC, Eisch AJ: Reduction of adult hippocampal neurogenesis confers vulnerability in an animal model of cocaine addiction. J Neurosci 30:304-315.

19. Levkowitz G, Zeller J, Sirotkin HI, French D, Schilbach S, Hashimoto H, Hibi M, Talbot WS, Rosenthal A: Zinc finger protein too few controls the development of monoaminergic neurons. Nat Neurosci 2003, 6:28-33.

20. Jeong JY, Einhorn Z, Mercurio S, Lee S, Lau B, Mione M, Wilson SW, Guo S: Neurogenin 1 is a determinant of zebrafish basal forebrain dopaminergic neurons and is regulated by the conserved zinc finger protein Tof/Fezl. Proc Natl Acad Sci USA 2006, 103:5143-5148.

21. Rink $E$, Guo $S$ : The too few mutant selectively affects subgroups of monoaminergic neurons in the zebrafish forebrain. Neuroscience 2004 127:147-154

22. Maldonado R, Saiardi A, Valverde O, Samad TA, Roques BP, Borrelli E: Absence of opiate rewarding effects in mice lacking dopamine D2 receptors. Nature 1997, 388:586-589.

23. Portavella M, Salas C, Vargas JP, Papini MR: Involvement of the telencephalon in spaced-trial avoidance learning in the goldfish (Carassius auratus). Physiol Behav 2003, 80:49-56.

24. Portavella M, Vargas JP, Torres B, Salas C: The effects of telencephalic pallial lesions on spatial, temporal, and emotional learning in goldfish. Brain Res Bull 2002, 57:397-399.

25. Rodriguez F, Duran E, Gomez A, Ocana FM, Alvarez E, Jimenez-Moya F, Broglio C, Salas C: Cognitive and emotional functions of the teleost fish cerebellum. Brain Res Bull 2005, 66:365-370.

26. Suboski MD, Bain S, Carty AE, McQuoid LM, Seelen MI, Seifert M: Alarm reaction in acquisition and social transmission of simulated-predator recognition by zebra Danio fish (Brachydanio rerio). J Comp Psych 1990, 104:101-112

27. Xu X, Scott-Scheiern T, Kempker L, Simons K: Active avoidance conditioning in zebrafish (Danio rerio). Neurobiol Learn Mem 2007, 87:72-77.

28. Blank M, Guerim LD, Cordeiro RF, Vianna MR: A one-trial inhibitory avoidance task to zebrafish: rapid acquisition of an NMDA-dependent long-term memory. Neurobiol Learn Mem 2009, 92:529-534.

29. Colwill RM, Raymond MP, Ferreira L, Escudero H: Visual discrimination learning in zebrafish (Danio rerio). Behav Processes 2005, 70:19-31.

30. Peitsaro N, Kaslin J, Anichtchik OV, Panula P: Modulation of the histaminergic system and behaviour by alpha-fluoromethylhistidine in zebrafish. J Neurochem 2003, 86:432-441.

31. Pather S, Gerlai R: Shuttle box learning in zebrafish (Danio rerio). Behav Brain Res 2009, 196:323-327.

32. Williams FE, White D, Messer WS: A simple spatial alternation task for assessing memory function in zebrafish. Behav Processes 2002, 58:125-132.

33. Ninkovic J, Folchert A, Makhankov YV, Neuhauss SC, Sillaber I, Straehle U, Bally-Cuif $\mathrm{L}$ : Genetic identification of $\mathrm{AChE}$ as a positive modulator of addiction to the psychostimulant D-amphetamine in zebrafish. J Neurobiol 2006, 66:463-475.

34. Levin ED, Bencan Z, Cerutti DT: Anxiolytic effects of nicotine in zebrafish. Physiol Behav 2007, 90:54-58.

35. Levin ED, Chen E: Nicotinic involvement in memory function in zebrafish. Neurotoxicol Teratol 2004, 26:731-735.

36. Wullimann MF, Mueller T: Identification and morphogenesis of the eminentia thalami in the zebrafish. J Comp Neurol 2004, 471:37-48.

37. Marks C, West TN, Bagatto B, Moore FB-G: Developmental environment alters conditional aggression in zebrafish. Copeia 2005, 4.

38. Rowland WJ: Studying visual cues in fish behaviour: a review of ethological techniques. Environmental Biology of Fishes 1999, 56:285-305.

39. Moretz JA, Martins EP, Robison BD: Behavioural syndromes and the evolution of correlated behavior in zebrafish. Behavioral Ecology 2007, 1-7.

40. Basquill SP, Grant JWA: An increase in habit complexity reduces aggression and monopolization of food by zebra fish (Danio rerio). Can $J$ Zool 1997, 76:770-772. 
41. Miklosi A, Andrew RJ: Right eye use associated with decision to bite in zebrafish. Behav Brain Res 1999, 105:199-205.

42. Popova NK: From gene to aggressive behavior: the role of brain serotonin. Neurosci Behav Physiol 2008, 38:471-475.

43. Nelson RJ: Biology of Aggression Oxford University Press 2006.

44. Colman JR, Baldwin D, Johnson LL, Scholz NL: Effects of the synthetic estrogen, 17alpha-ethinylestradiol, on aggression and courtship behavior in male zebrafish (Danio rerio). Aquat Toxicol 2009, 91:346-354.

45. Larson ET, O'Malley DM, Melloni RH Jr: Aggression and vasotocin are associated with dominant-subordinate relationships in zebrafish. Behav Brain Res 2006, 167:94-102.

46. Wai MS, Lorke DE, Webb SE, Yew DT: The pattern of c-fos activation in the CNS is related to behavior in the mudskipper, Periophthalmus cantonensis. Behav Brain Res 2006, 167:318-327.

47. Demski LS, Knigge KM: The telencephalon and hypothalamus of the bluegill (Lepomis macrochirus): evoked feeding, aggressive and reproductive behavior with representative frontal sections. J Comp Neurol 1971, 143:1-16.

48. Blaser RE, Chadwick L, McGinnis GC: Behavioral measures of anxiety in zebrafish (Danio rerio). Behav Brain Res 2009, 208:56-62.

49. Lopez-Patino MA, Yu L, Cabral H, Zhdanova IV: Anxiogenic effects of cocaine withdrawal in zebrafish. Physiol Behav 2008, 93:160-171.

50. Egan RJ, Bergner CL, Hart PC, Cachat JM, Canavello PR, Elegante MF, Elkhayat SI, Bartels BK, Tien AK, Tien DH, et al: Understanding behavioral and physiological phenotypes of stress and anxiety in zebrafish. Behav Brain Res 2009, 205:38-44.

51. Serra EL, Medalha CC, Mattioli R: Natural preference of zebrafish (Danio rerio) for a dark environment. Braz J Med Biol Res 1999, 32:1551-1553.

52. Speedie N, Gerlai R: Alarm substance induced behavioral responses in zebrafish (Danio rerio). Behav Brain Res 2008, 188:168-177.

53. Wong K, Elegante M, Bartels B, Elkhayat S, Tien D, Roy S, Goodspeed J, Suciu C, Tan J, Grimes C, et al: Analyzing habituation responses to novelty in zebrafish (Danio rerio). Behav Brain Res 2009, 208:450-457.

54. Levin ED, Limpuangthip J, Rachakonda T, Peterson M: Timing of nicotine effects on learning in zebrafish. Psychopharmacology (Berl) 2006, 184:547-552.

55. Bencan Z, Sledge D, Levin ED: Buspirone, chlordiazepoxide and diazepam effects in a zebrafish model of anxiety. Pharmacol Biochem Behav 2009, 94:75-80.

56. Zimmerman JE, Naidoo N, Raizen DM, Pack Al: Conservation of sleep: insights from non-mammalian model systems. Trends Neurosci 2008, 31:371-376.

57. Zhdanova IV: Sleep in zebrafish. Zebrafish 2006, 3:215-226.

58. Yokogawa T, Marin W, Faraco J, Pezeron G, Appelbaum L, Zhang J, Rosa F, Mourrain $\mathrm{P}$, Mignot $\mathrm{E}$ : Characterization of sleep in zebrafish and insomnia in hypocretin receptor mutants. PLOS Biol 2007, 5:e277.

59. Fleckenstein $A E$, Volz TJ, Riddle EL, Gibb JW, Hanson GR: New insights into the mechanism of action of amphetamines. Annu Rev Pharmacol Toxicol 2007, 47:681-698.

60. Harrison NL: Mechanisms of sleep induction by GABA(A) receptor agonists. J Clin Psychiatry 2007, 68(Suppl 5):6-12

61. Ruuskanen JO, Peitsaro N, Kaslin JV, Panula P, Scheinin M: Expression and function of alpha-adrenoceptors in zebrafish: drug effects, mRNA and receptor distributions. J Neurochem 2005, 94:1559-1569.

62. Renier C, Faraco JH, Bourgin P, Motley T, Bonaventure P, Rosa F, Mignot E: Genomic and functional conservation of sedative-hypnotic targets in the zebrafish. Pharmacogenet Genomics 2007, 17:237-253.

63. Appelbaum L, Wang GX, Maro GS, Mori R, Tovin A, Marin W, Yokogawa T, Kawakami K, Smith SJ, Gothilf Y, et al: Sleep-wake regulation and hypocretin-melatonin interaction in zebrafish. Proc Natl Acad Sci USA 2009, 106:21942-21947.

64. Bang PI, Yelick PC, Malicki JJ, Sewell WF: High-throughput behavioral screening method for detecting auditory response defects in zebrafish. $J$ Neurosci Methods 2002, 118:177-187.

65. Solnica-Krezel L, Schier AF, Driever W: Efficient recovery of ENU-induced mutations from the zebrafish germline. Genetics 1994, 136:1401-1420.

66. Amsterdam A, Hopkins N: Mutagenesis strategies in zebrafish for identifying genes involved in development and disease. Trends Genet 2006, 22:473-478.
67. Urasaki A, Kawakami K: Analysis of genes and genome by the tol2mediated gene and enhancer trap methods. Methods Mol Biol 2009, 546:85-102.

68. Petzold AM, Balciunas D, Sivasubbu S, Clark KJ, Bedell VM, Westcot SE, Myers SR, Moulder GL, Thomas MJ, Ekker SC: Nicotine response genetics in the zebrafish. Proc Natl Acad Sci USA 2009, 106:18662-18667.

69. Moens CB, Donn TM, Wolf-Saxon ER, Ma TP: Reverse genetics in zebrafish by TILLING. Brief Funct Genomic Proteomic 2008, 7:454-459.

70. Ekker SC: Zinc finger-based knockout punches for zebrafish genes. Zebrafish 2008, 5:121-123.

71. Blomme T, Vandepoele K, De Bodt S, Simillion C, Maere S, Van de Peer Y: The gain and loss of genes during 600 million years of vertebrate evolution. Genome Biol 2006, 7:R43.

72. Holland PW, Garcia-Fernandez J: Hox genes and chordate evolution. Dev Biol 1996, 173:382-395.

73. Yamaguchi A, Ishii H, Morita I, Oota I, Takeda H: mRNA expression of fibroblast growth factors and hepatocyte growth factor in rat plantaris muscle following denervation and compensatory overload. Pflugers Arch 2004, 448:539-546.

74. Deng CX, Wynshaw-Boris A, Shen MM, Daugherty C, Ornitz DM, Leder P: Murine FGFR-1 is required for early postimplantation growth and axial organization. Genes Dev 1994, 8:3045-3057.

75. Koide T, Miyasaka N, Morimoto K, Asakawa K, Urasaki A, Kawakami K, Yoshihara Y: Olfactory neural circuitry for attraction to amino acids revealed by transposon-mediated gene trap approach in zebrafish. Proc Natl Acad Sci USA 2009, 106:9884-9889.

76. Mueller KP, Neuhauss SC: Quantitative measurements of the optokinetic response in adult fish. J Neurosci Methods 2009, 186(1):29-34.

77. Miklosi A, Andrew RJ, Savage H: Behavioural lateralisation of the tetrapod type in the zebrafish (Brachydanio rerio). Physiol Behav 1997, 63:127-135.

78. Engeszer RE, Barbiano LA, Ryan MJ, Parichy DM: Timing and plasticity of shoaling behaviour in the zebrafish, Danio rerio. Anim Behav 2007, 74:1269-1275.

79. Engeszer RE, Wang G, Ryan MJ, Parichy DM: Sex-specific perceptual spaces for a vertebrate basal social aggregative behavior. Proc Natl Acad Sci USA 2008, 105:929-933.

80. Blin M, Norton W, Bally-Cuif L, Vernier P: NR4A2 controls the differentiation of selective dopaminergic nuclei in the zebrafish brain. Mol Cell Neurosci 2008, 39:592-604.

81. Fontaine E, Lentink D, Kranenbarg S, Muller UK, van Leeuwen JL, Barr AH, Burdick JW: Automated visual tracking for studying the ontogeny of zebrafish swimming. J Exp Biol 2008, 211:1305-1316.

82. Darrow KO, Harris WA: Characterization and development of courtship in zebrafish, Danio rerio. Zebrafish 2004, 1:40-45.

83. Gabriel JP, Mahmood R, Kyriakatos A, Soll I, Hauptmann G, Calabrese RL, El Manira A: Serotonergic modulation of locomotion in zebrafish: endogenous release and synaptic mechanisms. J Neurosci 2009, 29:10387-10395.

84. Arrenberg $A B$, Del Bene $F$, Baier $\mathrm{H}$ : Optical control of zebrafish behavior with halorhodopsin. Proc Natl Acad Sci USA 2009, 106:17968-17973.

85. Naumann EA, Kampff AR, Prober DA, Schier AF, Engert F: Monitoring neural activity with bioluminescence during natural behavior. Nat Neurosci 13:513-520.

86. Dlugos CA, Rabin RA: Ethanol effects on three strains of zebrafish: model system for genetic investigations. Pharmacol Biochem Behav 2003, 74:471-480.

87. Hall D, Suboski MD: Visual and olfactory stimuli in learned release of alarm reactions by zebra danio fish (Brachydanio rerio). Neurobiol Learn Mem 1995, 63:229-240.

88. Wright D, Rimmer LB, Pritchard VL, Krause J, Butlin RK: Inter and intrapopulation variation in shoaling and boldness in the zebrafish (Danio rerio). Naturwissenschaften 2003, 90:374-377.

89. Gerlai R: Zebra fish: an uncharted behavior genetic model. Behav Genet 2003, 33:461-468.

90. Swain HA, Sigstad C, Scalzo FM: Effects of dizocilpine (MK-801) on circling behavior, swimming activity, and place preference in zebrafish (Danio rerio). Neurotoxicol Teratol 2004, 26:725-729.

91. Anichtchik OV, Kaslin J, Peitsaro N, Scheinin M, Panula P: Neurochemical and behavioural changes in zebrafish Danio rerio after systemic 
administration of 6-hydroxydopamine and 1-methyl-4-phenyl-1,2,3,6tetrahydropyridine. J Neurochem 2004, 88:443-453.

92. Turnell ER, Mann KD, Rosenthal GG, Gerlach G: Mate choice in zebrafish (Danio rerio) analyzed with video-stimulus techniques. Biol Bull 2003, 205:225-226.

93. Pradel G, Schachner M, Schmidt R: Inhibition of memory consolidation by antibodies against cell adhesion molecules after active avoidance conditioning in zebrafish. J Neurobiol 1999, 39:197-206.

94. Pradel G, Schmidt R, Schachner M: Involvement of L1.1 in memory consolidation after active avoidance conditioning in zebrafish. $J$ Neurobiol 2000, 43:389-403.

doi:10.1186/1471-2202-11-90

Cite this article as: Norton and Bally-Cuif: Adult zebrafish as a model

organism for behavioural genetics. BMC Neuroscience 2010 11:90.

Submit your next manuscript to BioMed Central and take full advantage of:

- Convenient online submission

- Thorough peer review

- No space constraints or color figure charges

- Immediate publication on acceptance

- Inclusion in PubMed, CAS, Scopus and Google Scholar

- Research which is freely available for redistribution

Submit your manuscript at www.biomedcentral.com/submit 